\title{
Optimization of Multiband White-Light Illuminants for Specified Color Temperatures
}

\author{
Snjezana Soltic ${ }^{1}$ and Andrew Neil Chalmers ${ }^{2}$ \\ ${ }^{1}$ Manukau Institute of Technology, Private Bag 94006, Manukau 2241, New Zealand \\ ${ }^{2}$ Auckland University of Technology, Institute of Biomedical Technologies, Private Bag 92006, Auckland 1142, New Zealand \\ Correspondence should be addressed to Andrew Neil Chalmers; achalmer@aut.ac.nz
}

Received 21 January 2015; Accepted 19 April 2015

Academic Editor: Xian An Cao

Copyright (c) 2015 S. Soltic and A. N. Chalmers. This is an open access article distributed under the Creative Commons Attribution License, which permits unrestricted use, distribution, and reproduction in any medium, provided the original work is properly cited.

This paper describes an effective approach for the optimization of multiband spectra to produce prospective white-light spectra having specific color temperatures. The optimization process employs a genetic algorithm known as differential evolution, which aims to minimize the color rendering differences between a prospective white-light spectrum and its corresponding reference illuminant. Color rendering is assessed by calculating the CIEDE2000 color difference $\left(\Delta E_{00}\right)$ for 14 CIE test colors under the two sources. Optimized white-light spectra were matched to three CIE standard illuminants, that is, A ( $2856 \mathrm{~K})$, $\mathrm{D}_{50}(5003 \mathrm{~K})$, and $\mathrm{D}_{65}(6504 \mathrm{~K})$. Optimal solutions for three- and four-band 25 and $50 \mathrm{~nm}$ Gaussian spectra are presented and analyzed, together with mixed 4-LED spectra that were optimized in the same way. In all cases, the simulated sources were shown to provide color rendering of such quality that $\Delta E_{00 \mathrm{av}} \leq 2.24$ units. Such white-light sources would likely find wide acceptance in numerous lighting applications.

\section{Introduction}

It is now firmly established that, by tuning the spectral intensities of light-emitting diodes (LEDs) which emit different bands of radiation, a white-light spectrum characterized by good color rendering and efficacy can be designed. LED spectra have been simulated [1-4] and spectra of real LEDs digitized for use [4-8]. Typically, the color rendering properties of the white-light spectrum are expressed using the color rendering index (CRI) recommended in Commission Internationale de l'Eclairage (CIE) publication 13.3 [9], and efficacy in terms of the luminous efficacy of radiation which is the ratio of luminous flux to radiant flux (LER, $1 \mathrm{~m} / \mathrm{rad}-\mathrm{W}$ ).

The performance of any given mixture is governed by the number of bands [7] and their shape [10], as well as the peak emission wavelengths $\left(\lambda_{i}\right)$ and bandwidths $\left(\Delta_{i}\right)$ of each of the bands used $[5,6]$. Starting with a trichromatic mixture of "Red," "Green," and "Blue" bands, the color rendering can be improved by the addition of an "Amber" band. Any further additional band $(n>4)$ increases the complexity of the mixing process and the control of the white-point, with only marginal performance benefits [7]. It is shown that if the peak wavelengths and bandwidths of available LEDs could be freely manipulated, then it would be possible to produce three-LED sources with excellent white-light spectra [5].

Our earlier work used an approach that focused purely on the best achievable combination of CRI and LER. The correlated color temperature (CCT) was an uncontrolled variable in the process, and the random CCTs that resulted were predominantly in the $3000-4000 \mathrm{~K}$ range. It has become apparent, however, that lighting manufacturers are desirous of spectral designs for light sources that can achieve specific target CCT values [11]. The marketing of light sources is evidently based upon a primary specification of CCT, with CRI and LER (while still of high importance) coming as secondary parameters.

We have therefore designed a new optimization process which puts CCT at the center of the process and then 
proceeds to optimize color rendering while maintaining a close tolerance to the target CCT value. We selected three CCT values to illustrate the effectiveness of our process.

Our approach excludes the physical processes that are needed for the conversion of input energy (most often electrical) into radiant energy. Our focus is rather on the distribution of energy within the radiant spectrum since this determines both the color and efficacy properties of a spectrum. As in our earlier work the optimization process was based on the differential evolution algorithm, as described in Section 2, and it is now based on the minimization of color differences expressed in the CIEDE2000 equation [12], currently recommended for computation of perceived color differences between color pairs. The results are presented in Section 3. It is demonstrated that white-light spectra with a desired CCT and both good color rendering and efficacy are feasible using three or four bands.

\section{Method}

The optimization tool is a Matlab implementation of the population-based differential evolution (DE) algorithm [13] where a population of possible solutions is evaluated using a fitness function. The search for an optimal solution starts with a population of $P$ randomly created solution vectors $\left\{S_{v 1}, \ldots, S_{v j}, \ldots, S_{v P}\right\}$, each vector representing a candidate mixed light spectrum, where some characteristics of the individual bands in the candidate spectrum, for example, intensities, are randomized. As new solutions are created and evaluated, only the fitter solutions are moved to the next generation [7].

The basis of the selection process in our algorithm is the color difference of specific surface colors as they appear under the candidate spectrum and under the reference spectrum of the same CCT. In each new generation, the offspring solutions are evaluated using a fitness function $\left(f_{\text {fit }}\right)$ based on the color difference $\Delta E_{00}$ calculated using the CIEDE2000 color difference formula. Hence, the algorithm searches for a spectrum with the lowest color differences. The optimum solution is determined after having performed $G$ (typically 1000) cycles of the evaluations; that is, the best solution in cycle $G$ is accepted as the best white-light spectrum.

Thus, the intent is to minimize the average color difference $\Delta E_{00(\text { Avg })}$ for a set of color samples. The color samples (Table 1) are the 14 test samples specified in CIE13.3 [9]. Samples 1 to 8 have low to moderate chromatic saturation; Samples 9 to 12 represent saturated red, yellow, green, and blue; and Samples 13 and 14 represent light human complexion and green foliage, respectively. The CIE test color samples are used since they constitute a well known and widely used set.

After the completion of each DE run, the performance of each optimized solution is assessed using the CIE general color rendering index, $R_{a}$, determined as per [9] and the lowest color rendering value $R_{\min }$, together with the LER. Furthermore, the $\Delta E_{00 i}$ for each test sample is tabulated (Tables 2, 3, 4, and 5), together with the color differences decomposed into chromatic differences [14] in terms of
TABLE 1: The 14 CIE test colors [9].

\begin{tabular}{lcc}
\hline Number & Style name & Brief description \\
\hline 1 & $7.5 \mathrm{R} 6 / 4$ & Light grayish red \\
2 & $5 \mathrm{Y} 6 / 4$ & Dark grayish yellow \\
3 & $5 \mathrm{GY} 6 / 8$ & Strong yellow green \\
4 & $2.5 \mathrm{G} 6 / 6$ & Moderate yellowish green \\
5 & $10 \mathrm{BG} 6 / 4$ & Light bluish green \\
6 & $5 \mathrm{~PB} 6 / 8$ & Light blue \\
7 & $2.5 \mathrm{P} 6 / 8$ & Light violet \\
8 & $10 \mathrm{P} 6 / 8$ & Light reddish purple \\
9 & $4.5 \mathrm{R} 4 / 13$ & Strong red \\
10 & $5 \mathrm{Y} 8 / 10$ & Strong yellow \\
11 & $4.5 \mathrm{G} 5 / 8$ & Strong green \\
12 & $3 \mathrm{~PB} 3 / 11$ & Strong blue \\
13 & $5 \mathrm{YR} 8 / 4$ & Light yellowish pink \\
14 & $5 \mathrm{GY} 4 / 4$ & Moderate olive green \\
\hline
\end{tabular}

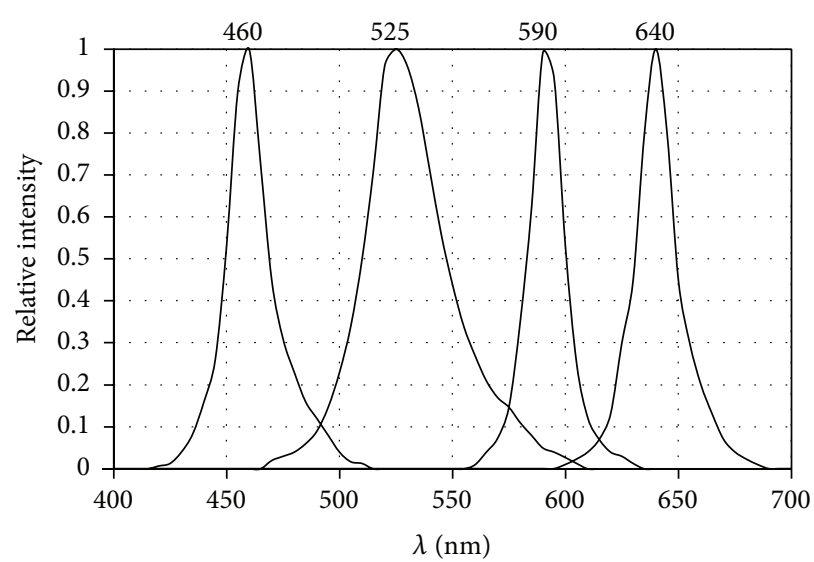

FIgURE 1: Spectral power distributions of real LEDs used in the optimized mixtures. The peak wavelengths $(\mathrm{nm})$ are given above each spectrum.

$\Delta C_{a b}{ }^{*}, \Delta H_{a b}{ }^{*}$, and $\Delta L^{*}$. Positive (negative) differences mean that the color test samples illuminated by the optimized spectrum have more (less) of that variable than when illuminated by the reference illuminant. The closeness of the chromaticity matches to the target CCTs has been computed in the CIE $\left(u^{\prime} v^{\prime}\right)$ chromaticity space [14]. These data are included on the grounds of the importance of this parameter to the potential users in the lighting industry. To gauge the significance of our computed $\Delta\left(u^{\prime} v^{\prime}\right)$ color differences, the color difference in the $\left(u^{\prime} v^{\prime}\right)$ diagram between $5000 \mathrm{~K}$ and $6500 \mathrm{~K}$ on the Planckian locus is $\Delta\left(u^{\prime} v^{\prime}\right) \sim 0.02$, and between $2700 \mathrm{~K}$ and $3000 \mathrm{~K}$ the difference is $\Delta\left(u^{\prime} v^{\prime}\right) \sim 0.01$.

2.1. Optimization of Real LED Mixtures. Here, a solution $\left(S_{v j}\right)$ is the spectrum of the light produced by a mixture of either 3 or 4 real LEDs, being a selected subset of the Luxeon range [15] (Figure 1). The optimization starts with a population of randomly created solution vectors, where the intensities $\left(I_{i}\right)$ of the individual LED spectra are randomized. The results are presented in Section 3.1. 
TABLE 2: Results: 4-LED optimization.

(a) Illuminant A: $R_{a}=93, R_{\min }=R_{11}=68$, LER $=3131 \mathrm{~lm} / \mathrm{rad}-\mathrm{W}$, $\Delta\left(u^{\prime} v^{\prime}\right)=0.0038$

\begin{tabular}{lccccc}
\hline Sample number & $\Delta E_{00}$ & $\Delta C_{a b}{ }^{*}$ & $\Delta H_{a b}{ }^{*}$ & $\Delta L^{*}$ & $R_{i}$ \\
\hline 1 & 0.58 & 0.55 & -0.62 & 0.23 & 95 \\
2 & 0.18 & 0.43 & 0.03 & 0.04 & 95 \\
3 & 0.65 & 0.01 & -0.10 & -0.76 & 96 \\
4 & 0.87 & 2.00 & 0.14 & 0.04 & 87 \\
5 & 0.47 & 0.89 & 0.16 & 0.05 & 90 \\
6 & 0.84 & 0.59 & 0.97 & 0.38 & 85 \\
7 & 0.40 & -0.15 & 0.46 & -0.06 & 94 \\
8 & 0.17 & -0.34 & -0.04 & -0.08 & 99 \\
9 & 0.41 & -0.55 & -0.70 & -0.08 & 99 \\
10 & 0.48 & -0.02 & 0.67 & -0.37 & 86 \\
11 & 2.45 & 5.61 & 2.01 & 0.58 & 68 \\
12 & 1.68 & 4.44 & -3.95 & 0.70 & 81 \\
13 & 0.31 & 0.54 & 0.20 & 0.17 & 91 \\
14 & 0.70 & -0.73 & -0.47 & -0.56 & 97 \\
Avg & 0.73 & - & - & - & 90 \\
\hline
\end{tabular}

(b) Illuminant $\mathrm{D}_{50}: R_{a}=95, R_{\min }=R_{12}=70, \mathrm{LER}=311 \mathrm{~lm} / \mathrm{rad}-\mathrm{W}$, $\Delta\left(u^{\prime} v^{\prime}\right)=0.0010$

\begin{tabular}{lccccc}
\hline Sample number & $\Delta E_{00}$ & $\Delta C_{a b}{ }^{*}$ & $\Delta H_{a b}{ }^{*}$ & $\Delta L^{*}$ & $R_{i}$ \\
\hline 1 & 0.56 & 0.75 & -0.53 & 0.17 & 94 \\
2 & 0.18 & 0.26 & 0.14 & 0.08 & 97 \\
3 & 0.61 & 0.55 & -0.75 & -0.42 & 95 \\
4 & 0.56 & 1.10 & -0.60 & 0.10 & 93 \\
5 & 0.50 & 0.61 & -0.55 & 0.04 & 95 \\
6 & 0.46 & 0.58 & 0.28 & 0.24 & 95 \\
7 & 0.47 & -0.79 & 0.44 & -0.12 & 95 \\
8 & 0.38 & -0.77 & -0.20 & -0.16 & 96 \\
9 & 0.44 & -0.52 & -0.7 & -0.22 & 96 \\
10 & 0.13 & -0.23 & 0.14 & -0.12 & 97 \\
11 & 1.67 & 4.53 & 0.90 & 0.57 & 79 \\
12 & 3.93 & 7.01 & -8.78 & 0.17 & 70 \\
13 & 0.70 & 0.55 & 0.73 & 0.12 & 92 \\
14 & 0.70 & -0.18 & -0.87 & -0.30 & 95 \\
Avg & 0.81 & - & - & - & 92 \\
\hline
\end{tabular}

(c) Illuminant $\mathrm{D}_{65}: R_{a}=95, R_{\min }=R_{12}=63, \mathrm{LER}=298 \mathrm{~lm} / \mathrm{rad}-\mathrm{W}$, $\Delta\left(u^{\prime} v^{\prime}\right)=0.0015$

\begin{tabular}{lccccc}
\hline Sample number & $\Delta E_{00}$ & $\Delta C_{a b}{ }^{*}$ & $\Delta H_{a b}{ }^{*}$ & $\Delta L^{*}$ & $R_{i}$ \\
\hline 1 & 0.58 & 0.80 & -0.52 & 0.10 & 94 \\
2 & 0.26 & 0.06 & 0.30 & 0.05 & 97 \\
3 & 0.59 & 0.62 & -0.83 & -0.34 & 95 \\
4 & 0.52 & 0.28 & -0.87 & 0.12 & 95 \\
5 & 0.71 & 0.36 & -0.93 & 0.07 & 95 \\
6 & 0.40 & 0.57 & 0.12 & 0.25 & 95 \\
7 & 0.66 & -1.36 & 0.49 & -0.10 & 93 \\
8 & 0.57 & -1.20 & -0.29 & -0.18 & 94 \\
9 & 0.49 & -0.40 & -0.73 & -0.32 & 95 \\
10 & 0.17 & -0.56 & 0.13 & -0.11 & 98 \\
11 & 1.38 & 3.80 & 0.59 & 0.56 & 85 \\
12 & 5.06 & 9.55 & -10.2 & 0.22 & 63 \\
13 & 1.02 & 0.43 & 1.08 & 0.05 & 92 \\
14 & 0.69 & -0.09 & -0.92 & -0.23 & 95 \\
Avg & 0.94 & - & - & - & 92 \\
\hline
\end{tabular}

TABLE 3: Results for Illuminant A simulations.

(a) $\mathrm{S}_{1}: R_{a}=84, R_{\min }=R_{12}=24, \mathrm{LER}=416 \mathrm{~lm} / \mathrm{rad}-\mathrm{W}, \Delta\left(u^{\prime} v^{\prime}\right)=0.0134$

\begin{tabular}{lccccc}
\hline Sample number & $\Delta E_{00}$ & $\Delta C_{a b}{ }^{*}$ & $\Delta H_{a b}{ }^{*}$ & $\Delta L^{*}$ & $R_{i}$ \\
\hline 1 & 1.64 & 2.13 & 1.49 & -0.45 & 93 \\
2 & 0.83 & 0.20 & 0.95 & -0.26 & 94 \\
3 & 3.49 & 6.65 & -3.48 & 2.03 & 49 \\
4 & 0.75 & 0.07 & -0.79 & 0.62 & 96 \\
5 & 0.99 & 1.05 & -1.27 & 0.17 & 87 \\
6 & 0.79 & 1.82 & -0.63 & -0.55 & 78 \\
7 & 0.76 & -1.51 & 0.04 & -0.30 & 96 \\
8 & 1.10 & -1.96 & -0.15 & -0.72 & 80 \\
9 & 1.71 & -2.90 & -2.24 & -1.01 & 26 \\
10 & 2.09 & 1.42 & -3.25 & 0.88 & 46 \\
11 & 1.94 & -2.48 & 2.56 & 0.44 & 73 \\
12 & 5.18 & 11.9 & -12.7 & -2.64 & 24 \\
13 & 2.74 & 0.27 & 3.26 & -0.92 & 93 \\
14 & 3.42 & 4.42 & -3.11 & 1.48 & 64 \\
Avg & 1.96 & - & - & - & 71 \\
\hline
\end{tabular}

(b) $\mathrm{S}_{2}: R_{a}=94, R_{\min }=R_{9}=70, \mathrm{LER}=357 \mathrm{~lm} / \mathrm{rad}-\mathrm{W}, \Delta\left(u^{\prime} v^{\prime}\right)=0.0054$

\begin{tabular}{lccccc}
\hline Sample number & $\Delta E_{00}$ & $\Delta C_{a b}{ }^{*}$ & $\Delta H_{a b}{ }^{*}$ & $\Delta L^{*}$ & $R_{i}$ \\
\hline 1 & 0.66 & 0.92 & 0.54 & -0.17 & 97 \\
2 & 0.38 & 0.02 & 0.45 & -0.08 & 98 \\
3 & 1.28 & 2.12 & -1.45 & 0.63 & 81 \\
4 & 0.39 & 0.18 & -0.49 & 0.25 & 99 \\
5 & 0.41 & 0.59 & -0.43 & 0.07 & 96 \\
6 & 0.50 & 0.96 & 0.21 & -0.20 & 95 \\
7 & 0.41 & -0.76 & 0.24 & -0.14 & 98 \\
8 & 0.48 & -0.92 & -0.15 & -0.25 & 91 \\
9 & 0.69 & -1.07 & -1.05 & -0.32 & 70 \\
10 & 0.65 & 0.19 & -1.01 & 0.28 & 80 \\
11 & 0.59 & -0.07 & 0.87 & 0.22 & 94 \\
12 & 2.10 & 5.38 & -4.97 & -0.93 & 71 \\
13 & 1.11 & 0.06 & 1.33 & -0.32 & 97 \\
14 & 1.41 & 1.41 & -1.44 & 0.45 & 85 \\
Avg & 0.79 & - & - & - & 89 \\
\hline
\end{tabular}

(c) $\mathrm{S}_{3}: R_{a}=96, R_{\min }=R_{12}=68, \mathrm{LER}=318 \mathrm{~lm} / \mathrm{rad}-\mathrm{W}, \Delta\left(u^{\prime} v^{\prime}\right)=0.0034$

\begin{tabular}{lccccc}
\hline Sample number & $\Delta E_{00}$ & $\Delta C_{a b}{ }^{*}$ & $\Delta H_{a b}{ }^{*}$ & $\Delta L^{*}$ & $R_{i}$ \\
\hline 1 & 0.59 & 0.52 & -0.68 & -0.00 & 96 \\
2 & 0.22 & 0.45 & 0.08 & 0.11 & 98 \\
3 & 0.66 & 1.37 & -0.72 & -0.13 & 90 \\
4 & 0.71 & -0.13 & -1.07 & 0.20 & 98 \\
5 & 0.93 & 0.78 & -1.27 & 0.09 & 96 \\
6 & 0.39 & 0.80 & 0.15 & 0.12 & 97 \\
7 & 0.57 & -0.97 & -0.00 & -0.39 & 97 \\
8 & 0.69 & -0.05 & -0.95 & -0.45 & 97 \\
9 & 0.85 & 1.13 & 0.42 & -0.79 & 97 \\
10 & 0.22 & -0.19 & -0.34 & 0.10 & 90 \\
11 & 1.48 & 2.61 & 1.58 & 0.44 & 89 \\
12 & 2.90 & 8.15 & -7.40 & -0.36 & 68 \\
13 & 0.39 & 0.35 & 0.42 & 0.10 & 99 \\
14 & 0.81 & 0.12 & -1.01 & -0.08 & 92 \\
Avg & 0.82 & - & - & - & 93 \\
\hline
\end{tabular}


TABLE 4: Results for Illuminant $\mathrm{D}_{50}$ simulations.

(a) $\mathrm{S}_{4}: R_{a}=85, R_{\min }=R_{12}=30, \mathrm{LER}=377 \mathrm{~lm} / \mathrm{rad}-\mathrm{W}, \Delta\left(u^{\prime} v^{\prime}\right)=0.0142$

\begin{tabular}{lccccc}
\hline Sample number & $\Delta E_{00}$ & $\Delta C_{a b}{ }^{*}$ & $\Delta H_{a b}{ }^{*}$ & $\Delta L^{*}$ & $R_{i}$ \\
\hline 1 & 2.13 & 3.27 & 1.56 & -0.35 & 90 \\
2 & 1.63 & -0.24 & 1.90 & -0.25 & 96 \\
3 & 3.04 & 5.92 & -3.24 & 1.85 & 58 \\
4 & 1.13 & -0.87 & -1.61 & 0.58 & 90 \\
5 & 1.23 & 1.30 & -1.47 & 0.15 & 87 \\
6 & 0.93 & 1.50 & 0.47 & -0.29 & 85 \\
7 & 1.14 & -2.52 & 0.36 & -0.24 & 90 \\
8 & 1.18 & -2.35 & -0.43 & -0.58 & 82 \\
9 & 1.55 & -1.89 & -2.65 & -0.41 & 36 \\
10 & 1.33 & 0.71 & -1.94 & 0.91 & 57 \\
11 & 1.45 & -2.03 & 1.91 & 0.65 & 76 \\
12 & 5.48 & 13.6 & -13.0 & -1.88 & 30 \\
13 & 3.82 & 1.03 & 4.40 & -0.87 & 88 \\
14 & 3.09 & 4.14 & -3.03 & 1.37 & 70 \\
Avg & 2.08 & - & - & - & 74 \\
\hline
\end{tabular}

(b) $\mathrm{S}_{5}: R_{a}=94, R_{\min }=R_{12}=75, \mathrm{LER}=323 \mathrm{~lm} / \mathrm{rad}-\mathrm{W}, \Delta\left(u^{\prime} v^{\prime}\right)=0.0050$

\begin{tabular}{lccccc}
\hline Sample number & $\Delta E_{00}$ & $\Delta C_{a b}{ }^{*}$ & $\Delta H_{a b}{ }^{*}$ & $\Delta L^{*}$ & $R_{i}$ \\
\hline 1 & 0.81 & 1.12 & 0.58 & -0.27 & 94 \\
2 & 0.84 & -0.63 & 0.91 & -0.23 & 97 \\
3 & 0.85 & 1.17 & -1.11 & 0.44 & 91 \\
4 & 0.97 & -1.06 & -1.45 & 0.22 & 92 \\
5 & 0.62 & 0.51 & -0.76 & 0.18 & 95 \\
6 & 0.46 & 0.33 & 0.47 & 0.11 & 98 \\
7 & 0.80 & -1.77 & -0.15 & 0.05 & 92 \\
8 & 0.73 & -0.96 & -0.99 & -0.07 & 93 \\
9 & 0.50 & 0.82 & -0.87 & -0.04 & 94 \\
10 & 0.24 & -1.00 & -0.09 & 0.01 & 94 \\
11 & 0.42 & -1.03 & -0.11 & 0.23 & 91 \\
12 & 2.41 & 5.08 & -5.68 & 0.09 & 75 \\
13 & 1.65 & -0.11 & 1.88 & -0.45 & 93 \\
14 & 0.97 & 0.91 & -1.10 & 0.35 & 92 \\
Avg & 0.88 & - & - & - & 92 \\
\hline
\end{tabular}

(c) $\mathrm{S}_{6}: R_{a}=95, R_{\min }=R_{12}=89, \mathrm{LER}=336 \mathrm{~lm} / \mathrm{rad}-\mathrm{W}, \Delta\left(u^{\prime} v^{\prime}\right)=0.0019$

\begin{tabular}{lccccc}
\hline Sample number & $\Delta E_{00}$ & $\Delta C_{a b}{ }^{*}$ & $\Delta H_{a b}{ }^{*}$ & $\Delta L^{*}$ & $R_{i}$ \\
\hline 1 & 0.55 & 0.68 & 0.48 & -0.09 & 94 \\
2 & 0.51 & -0.61 & 0.44 & -0.26 & 97 \\
3 & 0.57 & 0.83 & -0.54 & 0.45 & 95 \\
4 & 0.36 & 0.89 & 0.14 & 0.05 & 97 \\
5 & 0.36 & 0.30 & 0.40 & 0.05 & 98 \\
6 & 0.32 & 0.59 & 0.13 & -0.06 & 96 \\
7 & 0.29 & -0.35 & 0.22 & 0.22 & 97 \\
8 & 0.81 & -1.49 & 0.73 & 0.06 & 88 \\
9 & 1.33 & -3.56 & -1.85 & 0.07 & 64 \\
10 & 0.13 & -0.32 & 0.07 & -0.14 & 99 \\
11 & 0.10 & -0.15 & 0.15 & -0.00 & 98 \\
12 & 1.43 & 2.15 & -3.12 & 0.11 & 89 \\
13 & 0.92 & -0.43 & 1.00 & -0.40 & 96 \\
14 & 0.50 & 0.65 & -0.40 & 0.28 & 97 \\
Avg & 0.58 & - & - & - & 93 \\
\hline
\end{tabular}

TABLE 5: Results for Illuminant $\mathrm{D}_{65}$ simulations.

(a) $\mathrm{S}_{7}: R_{a}=87, R_{\min }=R_{12}=38, \mathrm{LER}=344 \mathrm{~lm} / \mathrm{rad}-\mathrm{W}, \Delta\left(u^{\prime} v^{\prime}\right)=0.0051$

\begin{tabular}{lccccc}
\hline Sample number & $\Delta E_{00}$ & $\Delta C_{a b}{ }^{*}$ & $\Delta H_{a b}{ }^{*}$ & $\Delta L^{*}$ & $R_{i}$ \\
\hline 1 & 1.86 & 2.94 & 1.07 & -0.15 & 83 \\
2 & 0.84 & -0.02 & 0.97 & -0.17 & 99 \\
3 & 3.22 & 6.71 & -3.96 & 1.49 & 58 \\
4 & 0.67 & 0.34 & -0.94 & 0.45 & 96 \\
5 & 1.16 & 0.64 & -1.51 & 0.06 & 92 \\
6 & 0.51 & 1.53 & -0.81 & -0.17 & 90 \\
7 & 0.73 & -1.68 & 0.24 & -0.16 & 94 \\
8 & 1.12 & -2.37 & 0.21 & -0.45 & 85 \\
9 & 2.45 & -4.15 & -3.96 & -0.02 & 42 \\
10 & 2.09 & 1.82 & -3.35 & 0.83 & 66 \\
11 & 1.58 & 0.31 & 2.57 & 0.75 & 86 \\
12 & 8.51 & 16.7 & -16.5 & -1.56 & 38 \\
13 & 3.43 & 0.79 & 3.76 & -0.68 & 83 \\
14 & 3.22 & 4.50 & -3.46 & 1.11 & 71 \\
Avg & 2.24 & - & - & - & 77 \\
\hline
\end{tabular}

(b) $\mathrm{S}_{8}: R_{a}=94, R_{\min }=R_{12}=69, \mathrm{LER}=315 \mathrm{~lm} / \mathrm{rad}-\mathrm{W}, \Delta\left(u^{\prime} v^{\prime}\right)=0.0053$

\begin{tabular}{lccccc}
\hline Sample number & $\Delta E_{00}$ & $\Delta C_{a b}{ }^{*}$ & $\Delta H_{a b}{ }^{*}$ & $\Delta L^{*}$ & $R_{i}$ \\
\hline 1 & 1.00 & 1.17 & 0.83 & -0.25 & 96 \\
2 & 0.17 & 0.01 & 0.17 & -0.09 & 96 \\
3 & 1.46 & 2.91 & -1.77 & 0.67 & 81 \\
4 & 0.53 & 1.09 & 0.09 & 0.39 & 97 \\
5 & 0.22 & 0.29 & -0.10 & 0.15 & 97 \\
6 & 0.26 & 0.69 & -0.45 & -0.04 & 93 \\
7 & 0.11 & 0.03 & -0.06 & -0.12 & 99 \\
8 & 0.19 & 0.07 & 0.07 & -0.22 & 97 \\
9 & 0.22 & -0.17 & -0.37 & -0.10 & 81 \\
10 & 0.98 & 0.85 & -1.59 & 0.30 & 80 \\
11 & 1.11 & 1.37 & 1.54 & 0.59 & 92 \\
12 & 4.02 & 6.17 & -7.7 & -0.68 & 69 \\
13 & 1.55 & 0.03 & 1.67 & -0.41 & 96 \\
14 & 1.46 & 1.95 & -1.54 & 0.50 & 87 \\
Avg & 0.95 & - & - & - & 90 \\
\hline
\end{tabular}

(c) $\mathrm{S}_{9}: R_{a}=93, R_{\min }=R_{9}=75, \mathrm{LER}=299 \mathrm{~lm} / \mathrm{rad}-\mathrm{W}, \Delta\left(u^{\prime} v^{\prime}\right)=0.0059$

\begin{tabular}{lccccc}
\hline Sample number & $\Delta E_{00}$ & $\Delta C_{a b}{ }^{*}$ & $\Delta H_{a b}{ }^{*}$ & $\Delta L^{*}$ & $R_{i}$ \\
\hline 1 & 0.26 & 0.13 & -0.31 & 0.07 & 93 \\
2 & 0.52 & -0.71 & 0.44 & -0.22 & 92 \\
3 & 0.28 & -0.38 & 0.33 & 0.20 & 95 \\
4 & 0.67 & -1.34 & -0.47 & -0.39 & 95 \\
5 & 0.37 & 0.01 & -0.43 & -0.19 & 94 \\
6 & 0.29 & 0.74 & -0.30 & -0.20 & 92 \\
7 & 0.63 & -0.89 & -0.35 & 0.47 & 95 \\
8 & 0.87 & -0.85 & -0.95 & 0.65 & 91 \\
9 & 2.17 & 0.50 & -2.96 & 1.62 & 75 \\
10 & 0.48 & -0.61 & 0.75 & -0.12 & 84 \\
11 & 1.59 & -3.45 & -1.10 & -0.88 & 88 \\
12 & 0.67 & 2.01 & -1.59 & 0.26 & 88 \\
13 & 0.34 & -0.66 & 0.04 & -0.15 & 95 \\
14 & 0.25 & -0.17 & 0.30 & 0.10 & 97 \\
Avg & 0.67 & - & - & - & 91 \\
\hline
\end{tabular}


2.2. Optimization of Synthetic LED Spectra Represented by Gaussian Functions. The above mentioned method is used with modification to optimize mixtures of Gaussian bands, where each band $\left(S_{i}\right)$ is simulated over a specific bandwidth within the wavelength range of $\lambda$ from 380 to $780 \mathrm{~nm}$ (in $1 \mathrm{~nm}$ increments). The properties of each Gaussian band are expressed in what follows:

$$
\begin{aligned}
S_{i}(\lambda) & =I_{i} e^{-\left(\lambda-\lambda_{i}\right)^{2} / 2 \delta^{2}}, \\
\delta & =\frac{\Delta_{i}}{2 \sqrt{2 \ln 2}},
\end{aligned}
$$

where $I_{i}, \lambda_{i}$, and $\Delta_{i}$ represent peak intensity, peak wavelength, and spectral bandwidth (or full-width at half-maximum, FWHM), respectively, and (2) represents the composite spectrum of $n$ bands, each having the same value of FWHM bandwidth:

$$
S_{v j}=\sum_{1}^{n} S_{i}(\lambda),
$$

with $n$ chosen to be either 3 or 4 as explained in the Section 3 .

The optimization starts with a population of randomly created solution vectors, where the intensities and the peak wavelengths of the individual Gaussian bands are randomized while their spectral bandwidths are kept constant. Two bandwidths have been investigated, $25 \mathrm{~nm}$ and $50 \mathrm{~nm}$, representing "typical” LED spectral bandwidths.

\section{Results}

3.1. Real LED Mixtures. We explored the feasibility of obtaining LED-based sources to match illuminants $A, D_{50}$, and $D_{65}$ using a set of real LEDs with the spectral power distributions shown in Figure 1. The choice was made to focus on the optimization of 4-band spectra as previous work $[3,5,7,8,10]$ indicated that mixing only the blue, green, and red would result in spectra with poor color rendering. An exception occurs if the red band can be broadened or the red peak wavelength lowered $[3,5]$.

The optimized 4-band spectra are shown in Figure 2. Table 2 shows that the spectra are acceptable standard illuminant simulators, having average color differences below $1 \Delta E_{00}$ unit, and CIE color rendering index $R_{a} \geq 93$. The $\Delta\left(u^{\prime} v^{\prime}\right)$ color differences are below 0.004 and could be considered subthreshold for white light.

Overall, the changes in lightness of the test colors are consistent regardless of the correlated color temperature; Samples 3, 7-10, and 14 become lighter and the remainder become darker (Table 2). This is thought to be due to the peaks and valleys in the combined spectra, resulting from the particular 4 LED spectra selected for the experiment.

The color differences are lowest for the Illuminant $\mathrm{A}$ simulation where 11 samples $(1-10,12-14)$ have both $\Delta C_{a b}{ }^{*}$ and $\Delta H_{a b}{ }^{*}$ below 5 units and the Sample 11 chroma difference is just above 5 units $\left(\Delta C_{a b}{ }^{*}{ }_{(11) \mathrm{A}}=5.61\right)$. For the Illuminant $\mathrm{A}$ simulations, the greatest change in hue is observed for Sample 12 (strong blue, $\Delta H_{a b}{ }^{*}{ }_{(12) \mathrm{A}}=-3.95$ ) and the highest change in lightness is observed for Sample 3 (strong yellow green) which becomes slightly darker $\left(\Delta L^{*}{ }_{(3) \mathrm{A}}=-0.76\right)$.

The Illuminant D simulations show significant increases in the color errors for Sample $12\left(\Delta C_{a b}{ }^{*}{ }_{(12) \mathrm{D} 50}=7.01\right.$, $\Delta H_{a b}{ }^{*}{ }_{(12) \mathrm{D} 50}=-8.78$, and $\Delta C_{a b}{ }^{*}{ }{ }^{12) \mathrm{D} 65}=9.55$, $\left.\Delta H_{a b}{ }^{*}(12) \mathrm{D} 65=-10.21\right)$.

3.2. Optimization of Gaussian Bands. Optimized spectra are shown in Figures 3, 4, and 5 with the values of the peak wavelengths given above each diagram.

3.2.1. Illuminant A. Figure 3 and Table 3 show the results of the optimization of the Illuminant A simulators showing that 3-band and 4-band Gaussian mixtures scored satisfactorily in the CRI metric $\left(R_{a} \geq 84\right)$. All simulators have a higher LER $(\geq 318 \mathrm{~lm} / \mathrm{rad}-\mathrm{W})$ than real Illuminant $\mathrm{A}\left(\mathrm{LER}_{\mathrm{A}}=156 \mathrm{~lm} / \mathrm{rad}-\right.$ $W)$. The spectra are named $S_{1}, S_{2}$, and $S_{3}$, where $S_{1}$ represents the $25 \mathrm{~nm} 3$-band spectrum, $S_{2}$ represents the $50 \mathrm{~nm} 3$-band spectrum, and $\mathrm{S}_{3}$ represents the $25 \mathrm{~nm} 4$-band spectrum. The $\Delta\left(u^{\prime} v^{\prime}\right)$ color differences are below 0.006 for $\mathrm{S}_{2}$ and $\mathrm{S}_{3}$.

As expected, the lower color errors (hence better color rendering) are obtained by either employing wider Gaussian bands $\left(\mathrm{S}_{2}\right)$ or using an additional 4 th band $\left(\mathrm{S}_{3}\right)$. However, a wider red band and the additional amber band reduced LER (from $416 \mathrm{~lm} / \mathrm{rad}-\mathrm{W}$ to 357 and $318 \mathrm{~lm} / \mathrm{rad}-\mathrm{W}$ ) by introducing more radiated power at wavelengths where the $V(\lambda)$ function has low values. The $25 \mathrm{~nm} 3$-band spectrum $\left(S_{1}\right)$ exhibits very poor rendering of blue (Sample 12: strong blue, $R_{12}=R_{\min }=$ 24) (Table 3(a)).

Simulators $S_{2}$ and $S_{3}$ render all color samples better than $S_{1}$, including the problematic saturated Samples 9-12. However, poor scores were recorded for Sample 9 (strong red) in $S_{2}$, and for Sample 12 (strong blue) in both $S_{2}$ and $S_{3}$.

Figure 3 shows the spectral power distributions of the Illuminant A simulations. The Gaussian peaks follow the general trend of the Illuminant A spectrum. The low emission in the blue region helps explain the problematic rendering of the strong blue sample shown in Table 3 .

Based on these results, the $S_{1}$ mixture would be an unsatisfactory simulator of Illuminant $A$, while the $S_{2}$ and $S_{3}$ mixtures would be acceptable for noncritical uses.

3.2.2. Illuminant $D_{50}$. The results for Illuminant $D_{50}$ are shown in Figure 4 and Table 4 . The spectra are named $\mathrm{S}_{4}$, $S_{5}$, and $S_{6}$, where $S_{4}$ represents the $25 \mathrm{~nm} \mathrm{3-band} \mathrm{spectrum,}$ $\mathrm{S}_{5}$ represents the $50 \mathrm{~nm}$ 3-band spectrum, and $S_{6}$ represents the $25 \mathrm{~nm}$ 4-band spectrum. The LER of the spectra is $\geq 323 \mathrm{~lm} / \mathrm{rad}-\mathrm{W}$ (versus the lower LER of real Illuminant $\mathrm{D}_{50}$ at $207 \mathrm{~lm} / \mathrm{rad}-\mathrm{W}$ ) and color rendering $R_{a} \geq 85$. Spectrum $\mathrm{S}_{4}$ has a particularly bad effect on the chroma and hue of the strong blue (Sample 12) $\left(\Delta C_{a b(12) S 4}=13.57, \Delta H_{a b}{ }^{*}{ }_{(12) S 4}=\right.$ $-12.96)$.

As expected, the color shifts and the differences in lightness are smaller for $S_{5}$ and $S_{6}$; that is, wider individual bands and 4-band mixture result in better white-light spectra. In particular, the best color rendering expressed in terms of $\Delta C_{a b}{ }^{*}$ and $\Delta H_{a b}{ }^{*}$ is for $\mathrm{S}_{6}$ where the color errors are all below 4 units. Also, $\mathrm{S}_{6}$ introduces the lowest changes 

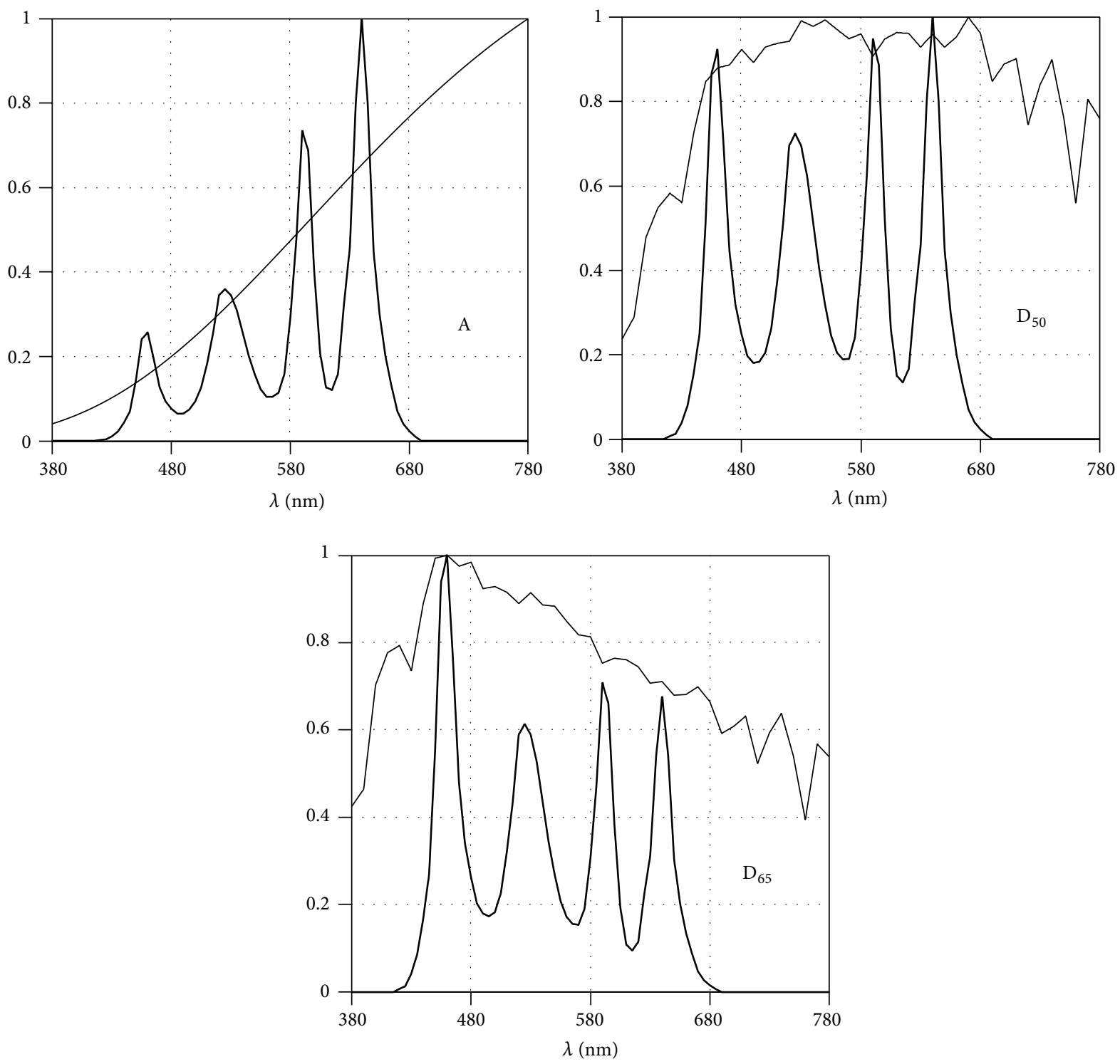

FIGURE 2: Optimized 4-LED relative spectral power distributions.

in lightness. Table 4 reveals considerable improvements in matching Illuminant $\mathrm{D}_{50}$ by widening the individual bands or by introducing the 4 th band. The $\Delta\left(u^{\prime} v^{\prime}\right)$ value of 0.0050 for $S_{5}$ is considered just-noticeable, and $\Delta\left(u^{\prime} v^{\prime}\right)=0.0019$ for $\mathrm{S}_{6}$ is below the noticeable threshold.

3.2.3. Illuminant $D_{65}$. Simulators for Illuminant $D_{65}$ (Figure 5 and Table 5) follow the same general trends as for $\mathrm{D}_{50}$, and the mixture with wider spectral bands $\left(\mathrm{S}_{8}\right)$ and the mixture with 4 bands $\left(\mathrm{S}_{9}\right)$ gave higher $R_{a}$ values and lower LER values than the $25 \mathrm{~nm} 3$-band mixture. The color rendering in terms of CIE CRI is highly satisfactory, $R_{a} \geq 87$, and the strong blue sample again exhibits the poorest color rendering $\left(R_{12(\mathrm{~S} 7)}=R_{\min }=38\right)$. The chromaticities of the simulations are very near to the chromaticity of Illuminant $\mathrm{D}_{65}, \Delta\left(u^{\prime} v^{\prime}\right)<0.006$ in all experiments. The LER of the spectra is $\geq 299 \mathrm{~lm} / \mathrm{rad}-\mathrm{W}$ (versus the LER of real Illuminant $\mathrm{D}_{65}$ at $\left.204 \mathrm{~lm} / \mathrm{rad}-\mathrm{W}\right)$.

Optimized spectrum $\mathrm{S}_{7}$ introduces larger color errors $\left(\Delta E_{00 \mathrm{av}}=2.24\right)$ than any other in this paper. In particular, the blue sample has both hue and chroma differences greater than 16 units (Table 5(a)). This aspect was somewhat unexpected and is thought to be due to the spectral discrepancies between the simulated spectra and real $\mathrm{D}_{65}$, particularly at the wavelength extremities. Further evidence is given by the fact that strong red (Sample 9) gives the next-worst $R_{i}$ in Table 5(a).

3.3. Comparison of Peak Wavelengths. It is instructive to compare our optimized Gaussian peak wavelengths with the peak wavelengths of the set of real LEDs, all of which are collated in Table 6. The real LEDs are labeled as "Blue" 

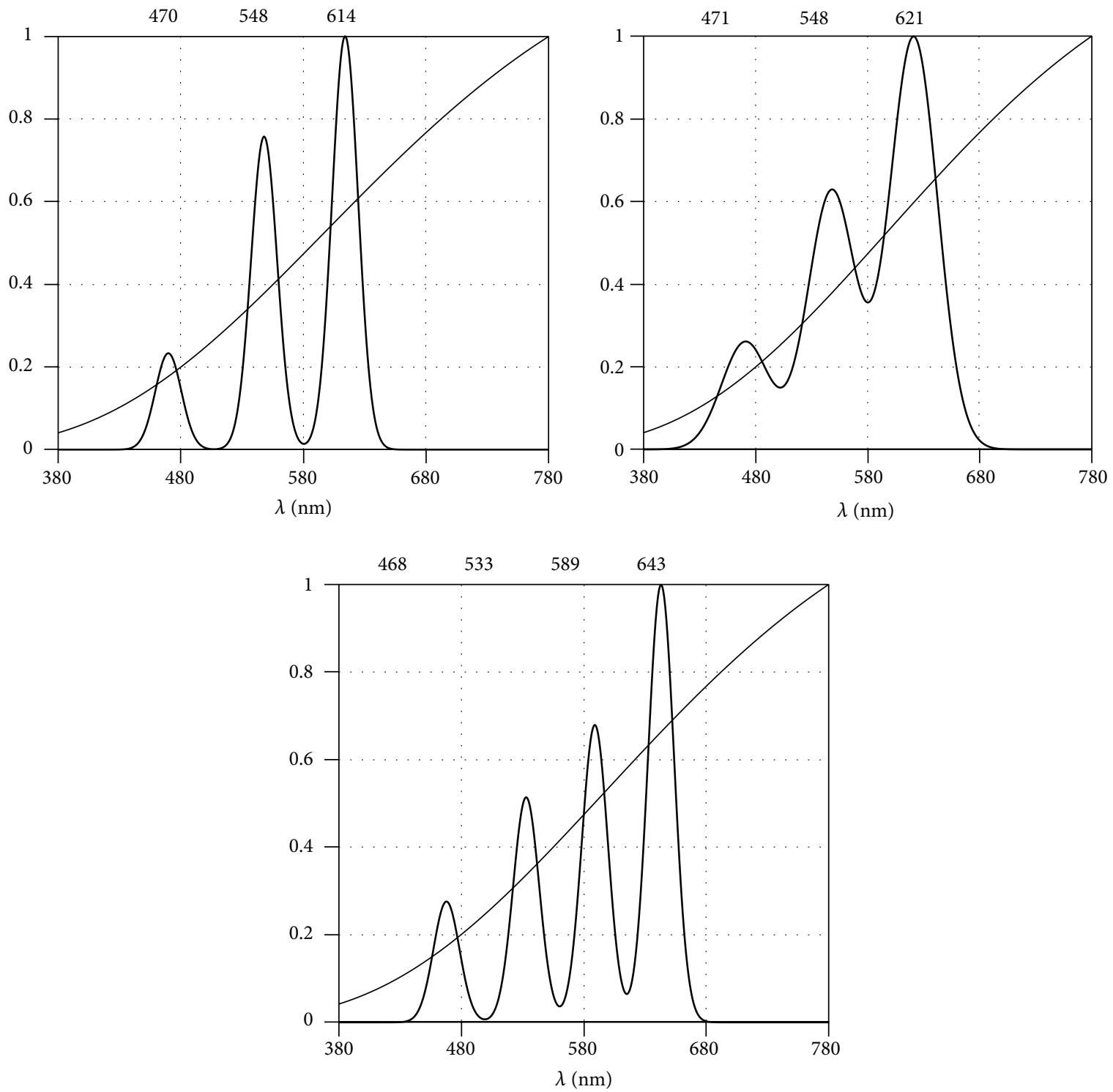

FIgURE 3: Optimized relative spectral power distributions $\left(\mathrm{S}_{1}, \mathrm{~S}_{2}, \mathrm{~S}_{3}\right)$ for Illuminant A.

TABLE 6: Comparison of optimized peak wavelengths.

\begin{tabular}{lccccc}
\hline Spectrum & $\begin{array}{c}\text { Real LEDs } \\
\text { Description }\end{array}$ & $\begin{array}{c}460 \mathrm{~nm} \\
\text { "Blue" }\end{array}$ & $\begin{array}{c}525 \mathrm{~nm} \\
\text { "Green" }\end{array}$ & $\begin{array}{c}590 \mathrm{~nm} \\
\text { "Amber" }\end{array}$ & $\begin{array}{c}640 \mathrm{~nm} \\
\text { "Red" }\end{array}$ \\
\hline $\mathrm{S}_{1}$ & $\mathrm{~A}, 25-\mathrm{nm}$ 3-band & 470 & 548 & - & 614 \\
$\mathrm{~S}_{2}$ & $\mathrm{~A}, 50-\mathrm{nm}$ 3-band & 471 & 548 & - & 621 \\
$\mathrm{~S}_{3}$ & $\mathrm{~A}, 25-\mathrm{nm}$ 4-band & 468 & 533 & 589 & 643 \\
$\mathrm{~S}_{4}$ & $\mathrm{D}_{50}, 25-\mathrm{nm}$ 3-band & 468 & 542 & - & 611 \\
$\mathrm{~S}_{5}$ & $\mathrm{D}_{50}, 50-\mathrm{nm}$ 3-band & 470 & 544 & - & 619 \\
$\mathrm{~S}_{6}$ & $\mathrm{D}_{50}, 25-\mathrm{nm}$ 4-band & 451 & 502 & 555 & 614 \\
$\mathrm{~S}_{7}$ & $\mathrm{D}_{65}, 25-\mathrm{nm}$ 3-band & 464 & 539 & - & 609 \\
$\mathrm{~S}_{8}$ & $\mathrm{D}_{65}, 50-\mathrm{nm}$ 3-band & 460 & 538 & - & 615 \\
$\mathrm{~S}_{9}$ & $\mathrm{D}_{65}, 25-\mathrm{nm}$ 4-band & 449 & 498 & 558 & 618 \\
\hline
\end{tabular}

$\left(\lambda_{B}=460 \mathrm{~nm}\right)$, "Green" $\left(\lambda_{G}=525 \mathrm{~nm}\right)$, "Amber" $\left(\lambda_{A}=\right.$ $590 \mathrm{~nm})$, and "Red" $\left(\lambda_{R}=640 \mathrm{~nm}\right)$.
In most of the spectra, the blue Gaussian band was between about $470 \mathrm{~nm}$ and $460 \mathrm{~nm}$. Exceptions are $S_{6}$ and $S_{9}$, the 4-band $\mathrm{D}_{50}$ and $\mathrm{D}_{65}$ simulators, in which the optimizer moved the blue and green bands to lower wavelengths to accommodate the amber band. The green peaks in the 3-band mixtures were optimized toward the peak of the $V_{\lambda}$ curve $(\lambda=555 \mathrm{~nm})$, thus producing the spectra with improved LER values as compared with the corresponding optimized LED mixtures.

It was consistently observed that the optimized wavelengths for the red band were between about $610 \mathrm{~nm}$ to $620 \mathrm{~nm}$. However, the 4-band Illuminant A simulator, $\mathrm{S}_{3}$, has the red band at $\lambda_{R(\mathrm{~S} 3)}=643 \mathrm{~nm}$. We ascribe that to the fact that the amber band was optimized to $\lambda_{A(S 3)}=589 \mathrm{~nm}$, as compared with $\lambda_{A(\mathrm{~S} 6)}=555 \mathrm{~nm}$ and $\lambda_{A(\mathrm{~S} 9)}=558 \mathrm{~nm}$ for the $\mathrm{D}$ simulators. The result was the noticeable improvement in the rendering of test Sample 9 with source $S_{3}$. 

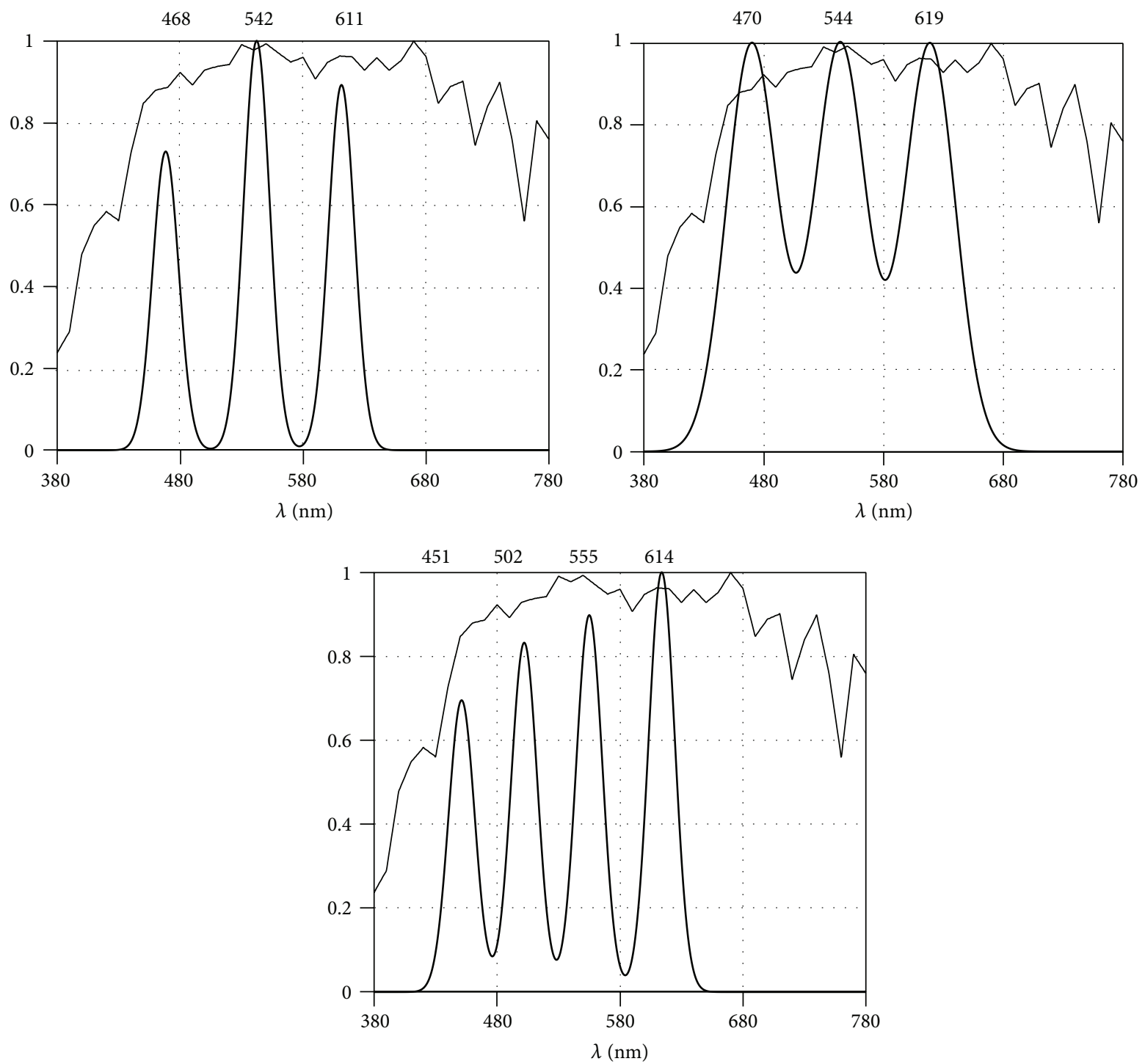

FIgURE 4: Optimized relative spectral power distributions $\left(S_{4}, S_{5}, S_{6}\right)$ for Illuminant $D_{50}$.

It was noteworthy in nearly all our results that the strong red (Sample 9) and strong blue (Sample 12) were badly affected by the truncation of the extrema of the synthesized spectra.

\section{Conclusions}

We have demonstrated that it is possible to simulate the CIE standard illuminants $\mathrm{A}, \mathrm{D}_{50}$, and $\mathrm{D}_{65}$ by mixing multiband LED and Gaussian spectra. The overall performance of the Gaussian-based mixtures was better than the LED-based counterparts due to the freedom to select the most suitable positions in the spectrum for the peak wavelengths in the Gaussian mixtures.

The simulation results show that 3-band Gaussian Illuminant A simulators could have CRI above 84 and LER double that of Illuminant A. Well designed 3-band Gaussian $\mathrm{D}_{50}$ and $\mathrm{D}_{65}$ simulators may have both CRI $\geq 85$ and LER $\geq$ $315 \mathrm{~lm} / \mathrm{rad}-\mathrm{W}$; 4-band simulators improve color rendering by distributing the bands in the visible spectrum.

Our optimization techniques, as described, do not need to be constrained by the choice of test color samples, color difference formulae, target spectrum, or choice of standard observer. This is demonstrated in this paper where we have shown how our previously published algorithm can be modified to apply new color difference techniques to new objectives, in this case the achievement of specific source CCT targets.

It should be noted that the spectra presented here are results of simulations, and further work is required to investigate the practical implementation of those spectra and to evaluate them in "real life" situations. 

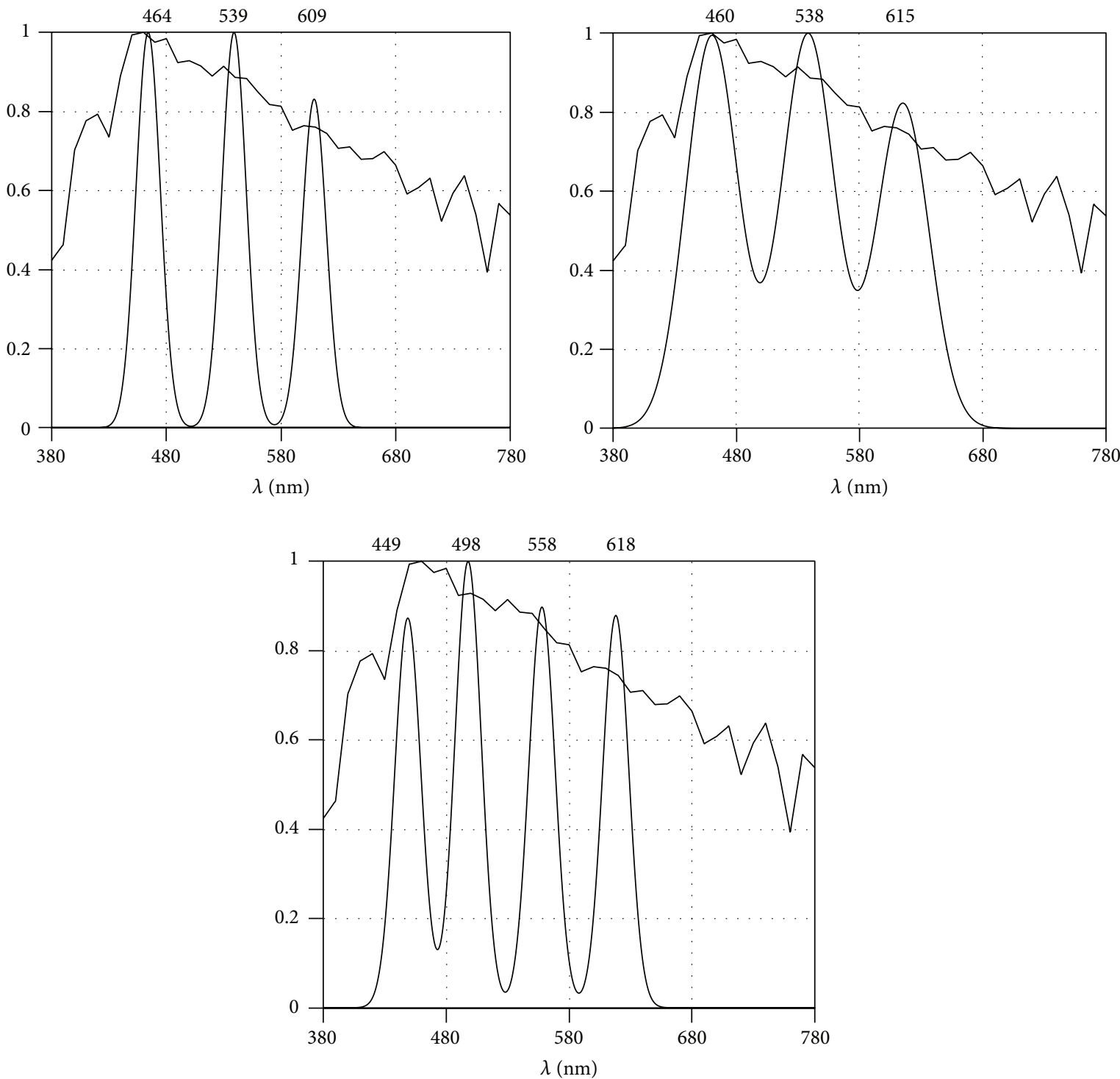

FIGURE 5: Optimized relative spectral power distributions $\left(S_{7}, S_{8}, S_{9}\right)$ for Illuminant $D_{65}$.

\section{Conflict of Interests}

The authors declare that there is no conflict of interests regarding the publication of this paper.

\section{Acknowledgment}

The authors wish to acknowledge the support provided for this work by the Technology Development Centre of the Manukau Institute of Technology.

\section{References}

[1] T. Erdem, S. Nizamoglu, X. W. Sun, and H. V. Demir, "A photometric investigation of ultra-efficient LEDs with high color rendering index and high luminous efficacy employing nanocrystal quantum dot luminophores," Optics Express, vol. 18, no. 1, pp. 340-347, 2010.
[2] G. He, L. Zheng, and H. Yan, "LED white lights with high CRI and high luminous efficacy," in LED and Display Technologies, vol. 7852 of Proceedings of SPIE, 2010.

[3] Y. Ohno, "Spectral design considerations for white LED color rendering," Optical Engineering, vol. 44, no. 11, Article ID 111302, 2005.

[4] A. Žukauskas, R. Vaicekauskas, F. Ivanauskas, R. Gaska, and M. S. Shur, "Optimization of white polychromatic semiconductor lamps," Applied Physics Letters, vol. 80, no. 2, pp. 234-236, 2002.

[5] E. Taylor, P. R. Edwards, and R. W. Martin, "Colorimetry and efficiency of white LEDs: spectral width dependence," Physica Status Solidi A: Applications and Materials Science, vol. 209, no. 3, pp. 461-464, 2012.

[6] R. S. Berns, "Designing white-light LED lighting for the display of art: a feasibility study," Color Research and Application, vol. 36, no. 5, pp. 324-334, 2011.

[7] S. Soltic and A. N. Chalmers, "Differential evolution for the optimisation of multi-band white LED light sources," Lighting Research \& Technology, vol. 44, no. 2, pp. 224-237, 2012. 
[8] A. Chalmers and S. Soltic, "Towards the optimum light source spectrum," Advances in OptoElectronics, vol. 2010, Article ID 596825, 9 pages, 2010.

[9] Commission Internationale de l'Eclairage, "Method of measuring and specifying color rendering properties of light sources," Tech. Rep. 13.3, CIE, Vienna, Austria, 1995.

[10] A. Chalmers and S. Soltic, "Light source optimization: spectral design and simulation of four-band white-light sources," Optical Engineering, vol. 51, no. 4, Article ID 044003, 2012.

[11] D. Sekulovski, Private Communication, Philips Lighting, 2012.

[12] Commission Internationale de l'Eclairage, "Improvement to industrial colour-difference evaluation," CIE Publication 142, CIE, Vienna, Austria, 2001.

[13] R. Storn and K. Price, "Differential evolution-a simple and efficient heuristic for global optimization over continuous spaces," Journal of Global Optimization, vol. 11, no. 4, pp. 341359, 1997.

[14] Commission Internationale de l'Eclairage, “Colorimetry," Tech. Rep. 15, CIE, Vienna, Austria, 2004.

[15] Lumileds Lighting, "Luxeon K2 Emitter," Technical Datasheet DS51, Lumileds Lighting, San Jose, Calif, USA, 2006. 

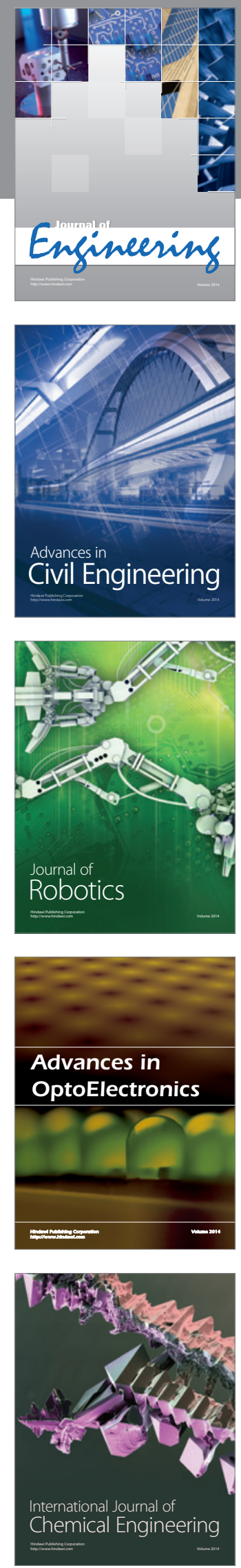

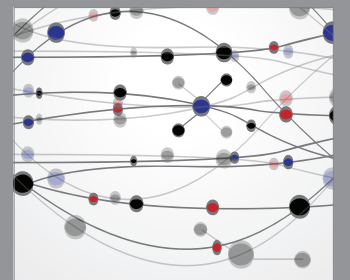

The Scientific World Journal
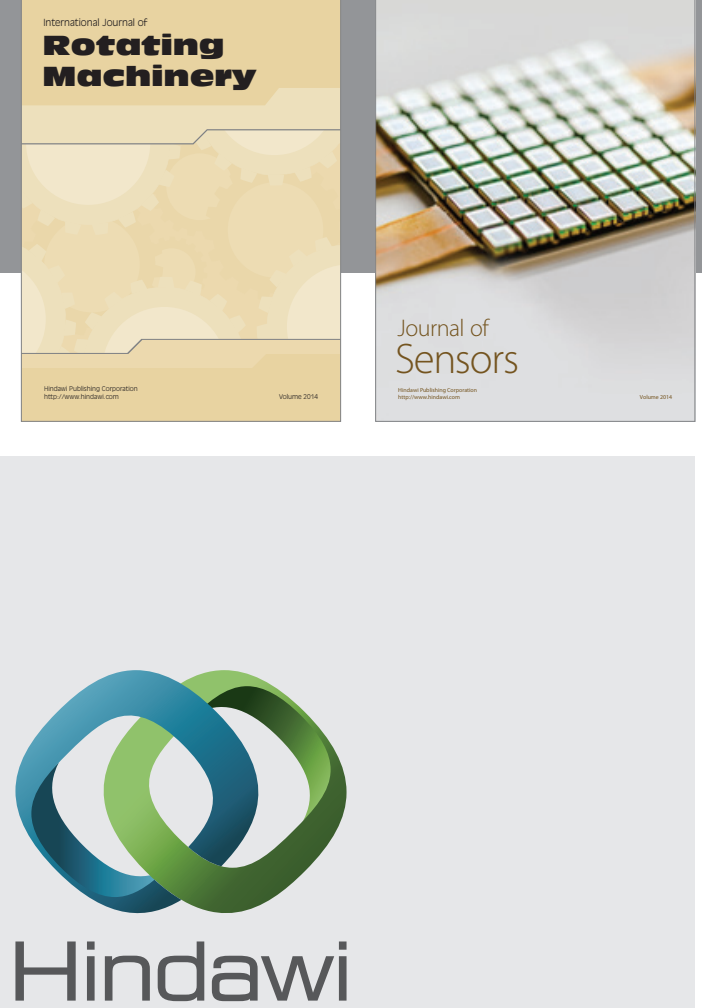

Submit your manuscripts at http://www.hindawi.com
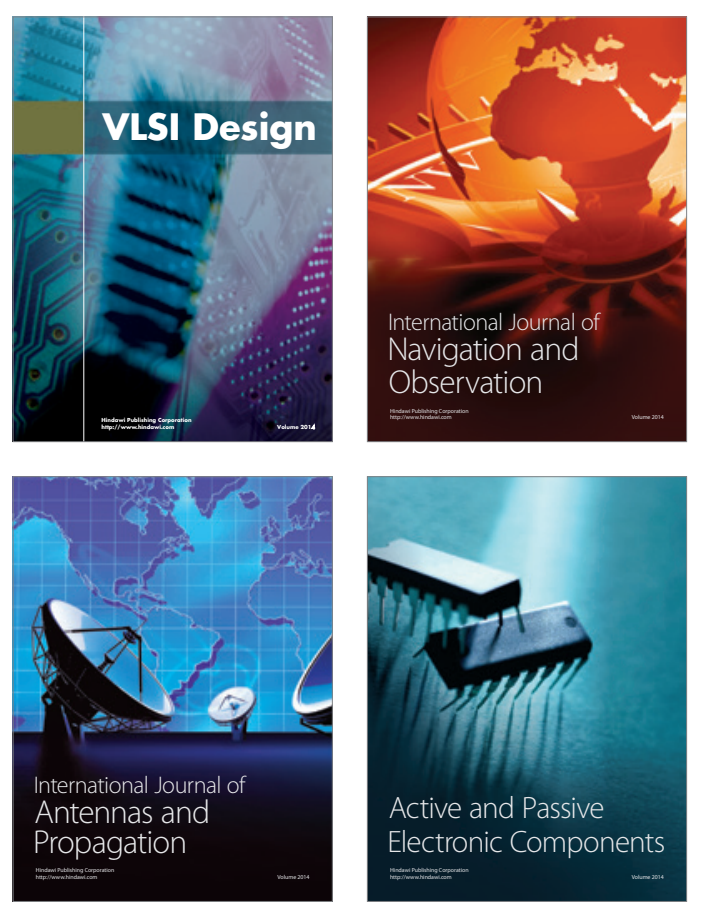
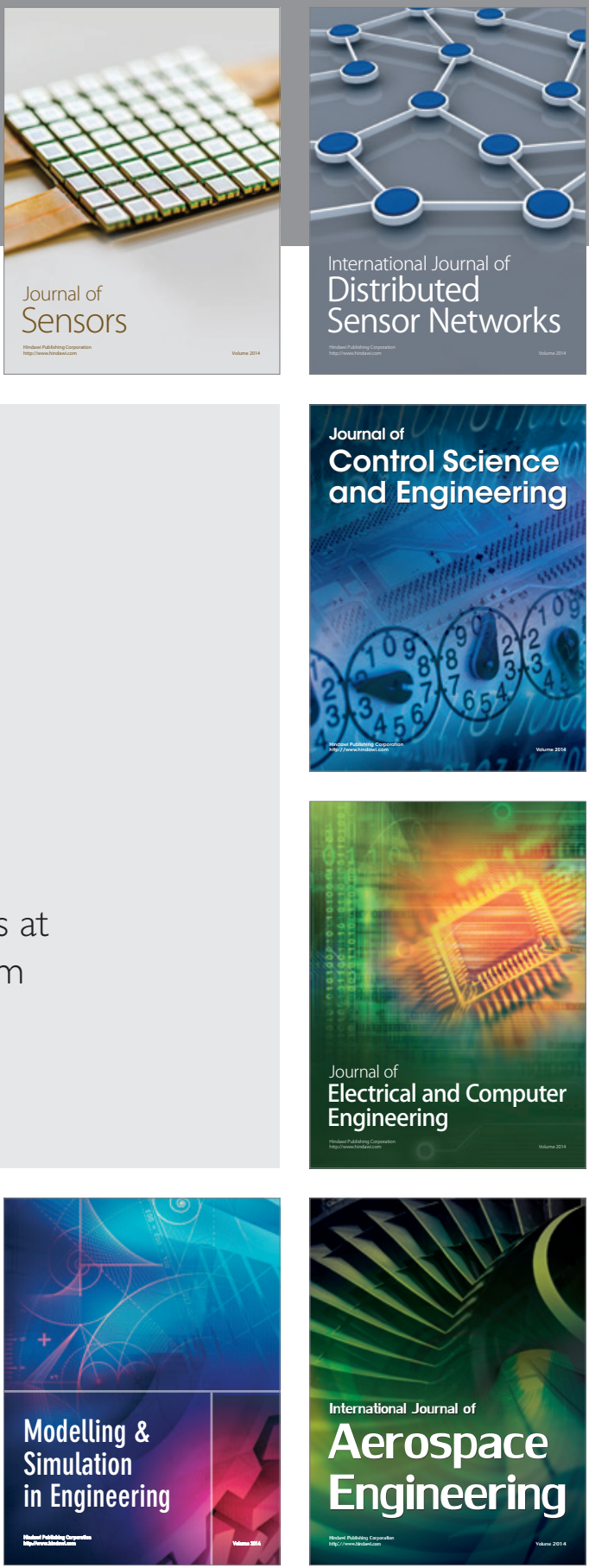

Journal of

Control Science

and Engineering
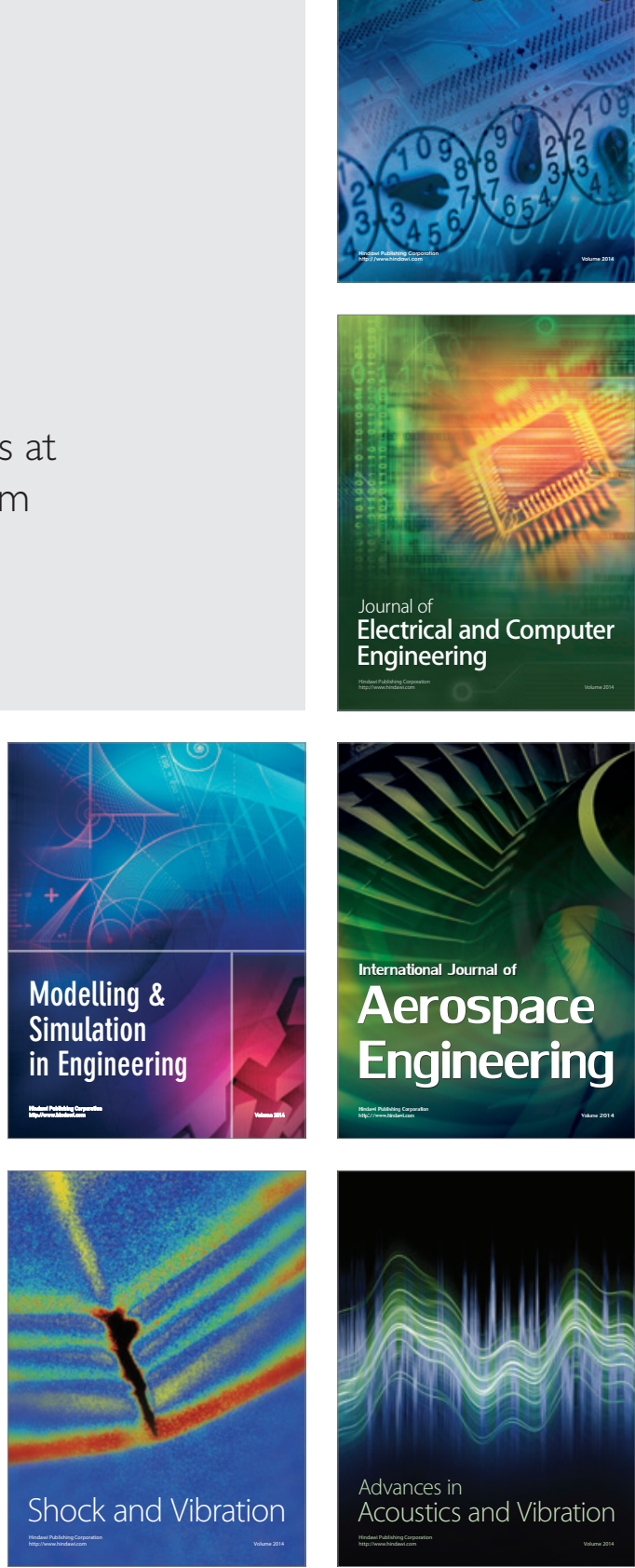